\title{
GENERALIZED EIGENVALUES OF AUTOMORPHISMS
}

\author{
M. A. AKCOGLU AND R. V. CHACON
}

The purpose of the present note is to construct a nonatomic measure space of finite measure $(S, \Sigma, \mu)$ and an automorphism $T$ such that, for any given $\omega \in F$, the equation

$$
f(T s)=\omega(s) \cdot f(s), \quad \text { for all } s \in S,
$$

has a solution $f \in F$, where $F$ is the class of complex-valued $\Sigma$-measurable functions of absolute value 1 . The question whether such an example exists was raised by Halmos [1, p. 97]. The construction yields a nonseparable measure space which with more care can be made to be just nonseparable. The problem remains open for separable measure spaces. It seems likely to us that no examples exist in the separable case.

Let $(I, Q, l)$ be the measure space obtained by setting $I=\{x \mid 0$ $\leqq x<1\}, \&$ the Borel sets of $I$, and $l$ the Lebesgue measure. We consider identical copies of this measure space, which are denoted by $\left(I_{j}, \mathbb{B}_{j}, l_{j}\right), j \in \Gamma$. The index set $\Gamma$ will be specified later. Let $(S, \Sigma, \mu)$ be defined by

$$
(S, \Sigma, \mu)=\prod_{j \in \Gamma}\left(I_{j}, \Theta_{j}, l_{j}\right) .
$$

We recall that $S$ is the Cartesian product space $\prod_{j \in \Gamma} I_{j}$ and hence the elements of $S$ are functions $s: \Gamma \rightarrow I$. The value of $s$ at $j \in \Gamma$ will be denoted by $s(j)$ and referred to as the $j$ th coordinate of $s$. We recall also that $\sigma$-field $\Sigma$ is generated by the class $\mathcal{G}$ of subsets $G$ of $S$ which have the form

$$
G=\prod_{j \in \Gamma} B_{j}
$$

where $B_{j} \in B_{j}$ for all $j \in \Gamma$ and $B_{j} \neq I_{j}$ for only finitely many $j$ 's, and that the measure $\mu$ is uniquely defined by setting

$$
\mu(G)=\prod_{j \in \Gamma} l_{j}\left(B_{j}\right)
$$

for $G$ in $\mathrm{g}$.

We let the index set $\Gamma$ be the initial segment $\{j \mid 1 \leqq j<J\}$ of ordinal numbers, ordered in the usual order, with upper limit $J$ defined as follows. For any set $M$, let $p(M)$ be its cardinal number. If $k$ is an ordinal number, let $p(k)=p(\{j \mid 1 \leqq j \leqq k\})$. We then let $J=\inf \{k \mid p(k)>c\}$ with $c=p(I)$.

Received by the editors May 8, 1964. 
Finally let $R$ be the class of all $\Sigma$-measurable functions $r: S \rightarrow I$.

Lemma 1. A function $r \in R$ can depend at most on a countable number of coordinates of $s$. For every $r \in R$, there exists an ordinal number $j_{r}<J$, such that $r$ does not depend on the coordinates $s(k)$ for $j_{r}<k<J$.

Proof. The first part of the lemma follows at once from $[2$, $\S 38(2)]$. The second part follows trivially on setting $j_{r}=\sup _{n} k_{n}$ where $k_{n}, n=1,2,3, \cdots$, are the coordinates on which $r$ depends. That $j_{r}<J$ follows since $p\left(k_{n}\right) \leqq c$.

TheOREM 1. There exists a one-to-one mapping $H$ of $\Gamma$ onto $R$ such that, for any $j \in \Gamma$, the function $r_{j}=H(j)$ does not depend on coordinates $s(k)$ for $j \leqq k<J$.

Proof. Let $R_{j}^{\prime}, 0 \leqq j<J$, be the subclass of $R$, consisting of functions which do not depend on $s(k)$ for $j<k<J$. Define

$$
R_{j}=R_{j}^{\prime}-\left(\bigcup_{0 \leqq k<j} R_{k}^{\prime}\right), \quad 0 \leqq j<J .
$$

By Lemma 1 and by this definition we have

$$
R=\underset{0 \leqq j<J}{\bigcup} R_{j}^{\prime}=\bigcup_{0 \leq j<J} R_{j} .
$$

The set $R_{0}$ consists of the constant functions $r: S \rightarrow I$. For $1 \leqq j<J$, $R_{j}$ contains those functions of $R_{j}^{\prime}$ which depend on $s(j)$. Hence we have $p\left(R_{j}\right) \geqq c, 0 \leqq j<J$. We now show that

$$
p\left(R_{j}\right)=c, \quad 0 \leqq j<J .
$$

For $j=0$ this assertion is obvious. To consider the other values of $j$, let

$$
\left(S_{j}, \Sigma_{j}, \mu_{j}\right)=\prod_{1 \leq k \leq j}\left(I_{k}, \bigotimes_{k}, l_{k}\right), \quad 1 \leqq j<J .
$$

Then $R_{j}^{\prime}$ can be considered as the class of all $\Sigma_{j}$-measurable functions $r: S_{j} \rightarrow I$. Let $\mathcal{G}_{j}$ be the generating class of $\Sigma_{j}$ as in (3). Since $p(B)=c$, it is easy to see that $p\left(\mathcal{G}_{j}\right)=c$, and thus also that $p\left(\Sigma_{j}\right)=c$. It follows therefore that $p\left(R_{j}^{\prime}\right)=c$, and thus (7) holds.

Now well-order every $R_{k}$, and note that this induces a lexicographic well-ordering of $R=\bigcup_{0 \leq k<J} R_{k}$. We denote this well-ordering by subindexing the elements of $R$, i.e., by $\left\{r_{1}, r_{2}, \cdots, r_{j}, \cdots\right\}$. More precisely, if $r_{j}=r^{k, u}$ and $r_{j^{\prime}}=r^{k^{\prime}, u^{\prime}}$, then $j \leqq j^{\prime}$ if and only if either

(a) $k<k^{\prime}$, or

(b) $k=k^{\prime}$ and $u \leqq u^{\prime}$, 
where $r^{k, u}$ is the $u$ th element of $R_{k}$ (and similarly for $r^{k^{\prime}, u^{\prime}}$ ). This wellordering of $R$ corresponds to the ordinal number $J$, i.e., $R=\left\{r_{j} \mid 1 \leqq j\right.$ $<J\}$. In fact, it is clear that $p(R)>c$, but $p\left(\cup_{0 \leq k \leq k_{0}} R_{k}\right)=c$ for any $k_{0}<J$. Hence $H(j)=r_{j}$ establishes a one-to-one mapping $H$ of $\Gamma$ onto $R$. To complete the proof of the theorem it is sufficient to show that, if $r_{j}=r^{k, u}$, then $j>k$. This follows, however, directly from the definitions.

THEOREM 2. Let the transformation $T: S \rightarrow S$ be defined by

$$
(T s)(j)=\left[s(j)+r_{j}(s)\right] \bmod 1, \quad \text { for all } j \in \Gamma, s \in S,
$$

where $r_{j}=H(j)$ as in Theorem 1 . Then $T$ is $\Sigma$-measurable and $\mu$-measure preserving, and for any $\omega \in F$ the equation $f(T s)=\omega(s) f(s)$ has a solution $f \in F$.

In order to prove this theorem we first note a few simple lemmas.

Lemma 2. Let $(W, \mathfrak{K})$ be a measurable space and let $f: W \rightarrow I$ be a $\mathfrak{K}$-measurable function. Then, for any $K \in \mathcal{K}$, the set

$$
\{(w, x) \mid w \in K, 0 \leqq x \leqq f(w)\}
$$

is $\mathcal{L}$-measurable in the product measure space

$$
(Y, \mathfrak{L})=(W, \mathfrak{K}) \times(I, \infty) .
$$

Proof. See $[2, \S 34(5)]$.

Lemma 3. Let $(W, \mathfrak{K}, \alpha)$ and $(Z, \Re, \gamma)$ be two finile measure spaces and let $Q: W \rightarrow Z$ be a transformation. Let $D$ be a generating class for the $\sigma$-field $\Re$, such that the class $\mathcal{E}$ of finite unions of disjoint sets from $D$ is a field. If $Q^{-1} D \in \Re$ and $\alpha\left(Q^{-1} D\right)=\gamma(D)$ for all $D \in D$, then $Q$ is $\Re-\Re$-measurable and $\alpha-\gamma$-measure preserving.

Proof. Consider the set

$$
\mathfrak{N}=\left\{M \mid M \in \Re, Q^{-1} M \in \mathfrak{K}, \alpha\left(Q^{-1} M\right)=\gamma(M)\right\} .
$$

It can be seen that $\mathscr{T}$ is a monotone class and contains the field $\varepsilon$. Therefore $\mathfrak{T}$ also contains the $\sigma$-field $\mathfrak{T}$ generated by $\mathcal{E}$.

Lemma 4. Consider a finite measure space $(W, \mathfrak{K}, \alpha)$ and let the transformation $P: W \rightarrow W$ be $K$-measurable and $\alpha$-measure preserving and let $f: W \rightarrow I$ be a $\mathcal{K}$-measurable function. Then the transformation $Q:(W \times I)$ $\rightarrow(W \times I)$ defined by

$$
Q(w, x)=(P w,[x+f(w)] \bmod 1)
$$

is $\mathcal{L}$-measurable and $\beta$-measure preserving in the product measure space 


$$
(Y, \mathcal{L}, \beta)=(W, \mathscr{K}, \alpha) \times(I, \propto, l) .
$$

Proof. Let $D$ be the class of sets $D$ of the form

$$
D=K \times B
$$

where $K \in \mathcal{K}$ and $B=\{x \mid a \leqq x<b\}, 0 \leqq a \leqq b \leqq 1$ is an interval of $I$. By virtue of Lemma 3 it is sufficient to prove that $D \in D$ implies $Q^{-1} D \in \mathscr{L}$ and $\beta\left(Q^{-1} D\right)=\beta(D)$. But

$$
Q^{-1}(K \times B)=\left\{(w, x) \mid w \in P^{-1} K, a \leqq[x+f(w)] \bmod 1<b\right\}
$$

and, applying Lemma 2 , we obtain that $Q^{-1}(K \times B)$ is $\mathscr{L}$-measurable. Furthermore

$$
\begin{aligned}
\beta\left(Q^{-1}(K \times B)\right) & =\int_{Q^{-1}(K \times B)} d \beta=\int_{P^{-1} K} \alpha(d w) \int_{a \leq[x+f(w)] \bmod 1<b} l(d x) \\
& =(b-a) \int_{P^{-1} K} \alpha(d w)=(b-a) \alpha\left(P^{-1} K\right) \\
& =(b-a) \alpha(K)=\alpha(K) l(B)=\beta(K \times B) .
\end{aligned}
$$

Proof of THEOREM 2. Let

$$
\left(S^{j}, \Sigma^{j}, \mu^{j}\right)=\prod_{1 \leqq k<j}\left(I_{k}, \Theta_{k}, l_{k}\right) .
$$

The transformation $T$ induces a set of transformations $T^{j}: S^{j} \rightarrow S^{j}$ such that

(11) $\left(T^{j} s^{j}\right)(k)=\left[s^{j}(k)+r_{k}\left(s^{j}\right)\right] \bmod 1, s^{j} \in S^{j}$, and $1 \leqq k<j$.

Note that we have made an obvious identification of the function $r_{k}: S \rightarrow I$ with a function $S^{i} \rightarrow I$, as we may, since $r_{k}$ does not depend, for $1 \leqq k \leqq j$, on the coordinates $s(u)$, for $k \leqq u<J$.

To any subset $M^{k}$ of $S^{k}$ associate a subset $M^{k^{*}}$ of $S$, by

$$
M^{k^{*}}=M^{k} \times\left[\prod_{k \leq k^{\prime}<J} I_{k^{\prime}}\right] .
$$

Then, for any $G \in \mathcal{G}$ there exists a $j<J$ and a set $G^{j}$ which belongs to the generating class $\mathcal{G}^{i}$ of $\Sigma^{i}$, such that $G=G^{*}$. Therefore we can write

$$
\mathcal{S}=\underset{2 \leqq j<J}{\bigcup} \mathcal{S}^{j^{*}}
$$

where

$$
\mathcal{G}^{*}=\left\{G^{j^{*}} \mid G^{j} \in \mathcal{G}^{j}\right\} .
$$

To prove that $T$ is $\Sigma$-measurable and $\mu$-measure preserving, it is 
sufficient to show that $G \in \mathcal{G}$ implies

$$
T^{-1} G \in \Sigma \text { and } \mu\left(T^{-1} G\right)=\mu(G) .
$$

To this end we apply a transfinite induction argument, considering relation (12). It is easy to see that the assertion is true for the sets of $\mathcal{G}^{2^{*}}$. Now assume that (13) holds for the sets of $\mathcal{G}^{j^{*}}, 2 \leqq j<j_{0}$, and consider the class $\mathcal{G}^{j 0^{*}}$. We separate two cases:

(a) The ordinal number $j_{0}$ has the immediate predecessor $k$, i.e., $j_{0}=k+1$. In this case, by virtue of Lemma 3 , the induction hypothesis is equivalent to the assumption that $T^{k}$ is a $\Sigma^{k}$-measurable and $\mu^{k}$-measure-preserving transformation. Since

$$
S^{j_{0}}=S^{k} \times I_{k}=\left\{\left(s^{k}, x_{k}\right) \mid s^{k} \in S^{k}, x_{k} \in I_{k}\right\}
$$

and

$$
T^{j_{0} j_{0}}=T^{j_{0}}\left(s^{k}, x_{k}\right)=\left(T^{k} s^{k},\left[x_{k}+r_{k}\left(s^{k}\right)\right] \bmod 1\right),
$$

Lemma 4 shows that $T^{j_{0}}$ is $\Sigma i_{0}$-measurable and $\mu^{j_{0}}$-measure preserving. Hence assertion (13) holds also for the sets of $g^{j 0^{*}}$.

(b) The ordinal number $j_{0}$ has no immediate predecessor. In this case we can see that

$$
\mathcal{G}^{j 0^{*}}=\underset{1 \leqq j<j_{0}}{\bigcup} \mathcal{G}^{j^{*}}
$$

We have defined then a nonatomic, finite measure space $(S, \Sigma, \mu)$ and a $\Sigma$-measurable, $\mu$-measure-preserving transformation $T$ (or automorphism): $S \rightarrow S$. To see that the equation (1) has a solution $f \in F$ for any given $\omega \in F$, note that there is a unique $r \in R$ such that $\omega(s)=e^{2 \pi i r(s)}$. Let $r=r_{j}$. Then $f(s)=e^{2 \pi i s(j)}$ is a solution of (1).

\section{REFERENCES}

1. P. R. Halmos, Lectures on ergodic theory, Publications of the Mathematical Society of Japan, no. 3, Math. Soc. Japan, Tokyo, 1956.

2. —, Measure theory, Van Nostrand, New York, 1950.

UNIVERSITY OF TORONTO AND

The Ohio State University 